\title{
Exploitation of Heterosis in Pearl Millet [Pennisetum glaucum (L.) R. Br.] for Yield and its Component Traits by Using Male Sterile Line
}

\author{
Zeal R. Acharya*, M.D. Khanapara, V.B. Chaudhari and Jalpa D. Dobaria
}

Department of Genetics and Plant Breeding, College of Agriculture, Junagdh Agricultural

University, Junagdh, 362001, India

*Corresponding author

\author{
A B S T R A C T
}

An experiment was conducted to study the mean performance and magnitude of heterosis of grain yield per plant and its thirteen yield attributing components in bajara. Experimental material consisting of 60 entries comprised of five male sterile lines and nine lines as well as their 45 hybrids developed through line $\mathrm{x}$ tester mating design along with standard check hybrid (GHB 732) were evaluated in a randomized block design with three replications. Perusal of mean data revealed that among females, JMSA 20102 and ICMA 04999 as well as among males, J-2508, J-2503 and J-2433 exhibited higher per se performance for grain yield per plant and its contributing traits. Considering per se performance of hybrids, the superior cross combinations for grain yield per plant were JMSA 20102 x J-2496 (19.56 g), JMSA 20102 x J-2479 (18.13 g) and JMSA 20102 x J$2500(15.53 \mathrm{~g})$. These cross combinations also had high per se performance for one or more yield contributing traits. Out of twenty eight significant heterotic cross combinations over better parents, nineteen showed positive and significant standard heterosis for grain yield per plant. Thus, the magnitude of heterosis toward the positive direction. The observed range of variation for grain yield per plant in hybrids was varied from 6.63g (ICMA 04999 x J-2510) to $19.56 \mathrm{~g}$ (JMSA $20102 \mathrm{x} \mathrm{J}-2496)$. The general mean of hybrids (10.24 g) and parents $(8.27 \mathrm{~g})$ indicated that the hybrids gave higher grain yield as compared to the parents.

\section{Introduction}

Pearl millet (Pennisetum glaucum (L.). R.Br.) is annual, tillering diploid $(2 \mathrm{n}=14)$ crop. It belongs to family Poaceae and sub family Paniceidae and believed to be originated in Africa. Pearl millet is one of the important staple food crops of India ranking fourth in acreage next to rice, wheat, and sorghum and world's sixth important cereal food crop. However, it is primarily grown as forage crop in USA, Australia and South Africa. It is a highly cross pollinated crop with protogynous flowering and wind borne pollination mechanism. Being a $\mathrm{C}_{4}$ species, it is endowed with a very high photosynthetic efficiency and more ability for dry matter production. Pearl millet is not only a quick growing short duration crop, but also found drought as well as heat tolerant and well adapted to different soil types. Because of its propensity for high dry matter production at high temperature, it has made a mark in tropics and sub-tropics. It is a drought resistant cereal having the 
maximum potentiality of grain production in adverse conditions. The better nutritive value of pearl millet grains appear from its protein, fat and mineral matters contents. It is also rich in vitamin $\mathrm{A}$, vitamin $\mathrm{B}$, thiamin as well as riboflavin contents and imparts substantial energy to the body with easy digestibility (Pal et al., 1996). Apart from grain, it also supplies fair quality of dry matter (forage and stover) at harvest in large bulk; which is an important secondary product in low resource agriculture for animal feed. The improvement in this crop in India started as early as in 1920. However, the real breakthrough was made when the first and the most widely used cytoplasmic male sterile line Tift 23A (Burton, 1965) was utilized in development of grain hybrids in India. Extensive testing of single crosses with $23 \mathrm{~A}_{1}$, Indian breeders could able to announce the release of 'HB-1' hybrid in 1965 (Athwal, 1965). Subsequently, availability of several cytoplasmic genetic male sterility systems have facilitated production and release of number of hybrids with increased drought tolerance as well as resistance to biotic stress and increased yield with greater efficiency in growth factor use (Burton, 1983; Andrews and Anand, 1992).

\section{Materials and Methods}

Experimental material consisting of 60 entries comprised of five male sterile lines (used as testers/females JSMA 101, JSMA 20102, ICMA 841, ICMA 89111, ICMA 04999) and nine inbred lines (used as lines/males J-2433, $\mathrm{J}-2479$ ，J-2482，J-2500，J-2503，J-2507，J$2508, \mathrm{~J}-2510)$ and their 45 hybrids developed through line $\mathrm{x}$ tester mating design along with standard check hybrid (GHB 732) were evaluated in a randomized block design with three replications. The 45 crosses made in line $\mathrm{x}$ tester mating design during summer 2016 at Instructional Farm, College of Agriculture, JAU, Junagadh, which were evaluated during kharif 2016 at the Sagdividi Farm,
Department of Seed Science and Technology, College of Agriculture, Junagadh Agricultural University, Junagadh. The data were recorded on five randomly selected plants per plot for each replications for the characters, viz., grain yield per plant, number of nodes on main stem, number of effective tillers per plant, plant height, ear head length, earhead girth, ear head weight, test weight, panicle index, total biomass per plant and harvest index. However, days to $50 \%$ flowering and days to maturity were recorded on plot basis.

The analysis of variance for experimental design was performed to test the significance of difference among the genotypes for the entire characters model as suggested by Panse and Sukhatme (1985). Heterobeltiosis was estimated as per the procedure given by Fonseca and Patterson (1968) using mean values for various characters over replications. Standard heterosis referred as the superiority of F1 over standard hybrid GHB 732 and was estimated as per the formula given by Meredith and Bridge (1972) for various characters over replications.

\section{Results and Discussion}

Considering per se performance of hybrids, more than 30 hybrids yielded higher than GHB 732 for seed yield per plant, of which two hybridsJMSA 20102 x J-2479 (18.13 g) and JMSA $20102 \times \mathrm{J}-2500$ (15.53 g) yielded significantly higher than GHB 732.Five superior cross combinations for seed yield per plant placed in Table 1 based on per se performance. Among them, three cross combinations JMSA 20102 x J-2496, JMSA $20102 \times \mathrm{J}-2479$ and JMSA $20102 \times \mathrm{J}-2500$ had high per se performance along with significant heterobeltiosis and standard heterosis for seed yield per plant. These crosses also manifested the significant and desirable heterotic effects for one or more yield contributing traits (Table 1). 
In the present study, heterosis over better parent (heterobeltiosis) and over standard check, GHB 732 (standard heterosis) was estimated. Several crosses exhibited conspicuous level of heterobeltiosis and standard heterosis for different characters are presented in Table 2 to 6 . Range of heterosis as well as number of crosses exhibited significance positive as well as negative heterobeltiosis and standard heterosis are presented in Table 2 to 6 .

With respect to the performance of hybrids for grain yield per plant, it was observed that twenty eight recorded significant heterobeltiosis, out of these, 19 hybrids had significant positive heterobeltiotic effect over standard check (GHB 732). The range of heterosis over better parent was from -41.93 to 112.68 per cent, while over standard check, it ranged from -23.17 to 112.60 per cent.

The cross JSMA $20102 \times$ J-2496 and depicted the significantly the highest and positive heterobeltiosis $(112.68 \%)$, standard heterosis $(126.60 \%)$ as well as the highest seed yield per plant. JMSA $20102 \times \mathrm{J}-2479$ and JMSA $20102 \times \mathrm{J}-2500$ were the next two best crosses exhibited significant and positive heterobeltiosis $(97.10 \%$ and $68.84 \%$, respectively), standard heterosis (110.04\% and $79.92 \%$, respectively). In such cases, expression of heterotic response over better and standard parents indicated the real superiority of hybrids from the commercial point of view. The high magnitude of heterobeltiosis was also observed by for other trait in pearl millet Aruselvi et al., (2006), Umaretiya (2006), Patel (2008), Vetriventhan et al., (2008), Shah (2009), Vagadiya et al., (2010), Patel (2012), Jethva et al., (2012) and Patel (2014).The medium magnitude of heterobeltiosis and standard heterosis were noticed for number of effective tillers per plant, plant height, ear head length, ear head weight and test weight. The medium magnitude of heterobeltiosis for these traits were also reported by Ramamoorthi and Govindarasu (2006), Aruselvi et al., (2008), Patel (2008), Vetriventhan et al., (2008), Shah (2009), Jethva et al., (2012), Patel (2012) and Patel (2014).

Table.1 Promising hybrids for seed yield per plant with standard heterosis and heterobeltiosis and component traits showing significant desirable standard heterosis

\begin{tabular}{||l|l|l|l|l|l||}
\hline \multirow{2}{*}{ Heterotic crosses } & \multirow{2}{*}{$\begin{array}{l}\text { Grain } \\
\text { yield } \\
\text { per } \\
\text { plant }(\mathbf{g})\end{array}$} & $\begin{array}{l}\text { Per cent heterosis of grain } \\
\text { yield per plant over }\end{array}$ & $\begin{array}{l}\text { Desirable heterosis for } \\
\text { other traits over }\end{array}$ \\
\cline { 3 - 6 } & $\begin{array}{l}\text { standard } \\
\text { heterosis }\end{array}$ & Heterobeltiosis & $\begin{array}{l}\text { Better } \\
\text { parent }\end{array}$ & $\begin{array}{l}\text { Check (GHB } \\
\mathbf{7 3 2})\end{array}$ \\
\hline JMSA 20102 x J-2496 & 19.56 & $126.60 * *$ & $112.68 * *$ & 3,9 & $1,2,3,4,5,6,8$ \\
\hline JMSA 20102 x J-2479 & 18.13 & $110.04 * *$ & $97.10^{* *}$ & 3,9 & $1,2,3,4,5,6,8$ \\
\hline JMSA 20102 x J-2500 & 15.53 & $79.92 * *$ & $68.84 * *$ & $1,3,8$ & $1,2,3,4,6,8$ \\
\hline JMSA 101 x J-2503 & 13.60 & $57.57 * *$ & $18.26 * *$ & $1,3,5,6,7,8$ & $1,3,4,5,6,7,8$ \\
\hline ICMA 89111 x J-2433 & 12.70 & $47.14 * *$ & $33.68 * *$ & $1,3,5,8$ & $1,2,3,4,5,6,7,8$ \\
\hline
\end{tabular}

*, ** Significant at 5 per cent and 1 per cent levels of significance, respectively

\begin{tabular}{|c|c|c|c|c|}
\hline$=$ & Numbers of nodes on main stem & 6 & $=$ & Test weight (g) \\
\hline$=$ & Numbers of effective tillers per plant & 7 & $=$ & Panicle index $(\%)$ \\
\hline$=$ & Plant height $(\mathrm{cm})$ & 8 & $=$ & Total biomass per plant (g) \\
\hline$=$ & Earhead girth $(\mathrm{cm})$ & 9 & $=$ & Harvest Index \\
\hline
\end{tabular}


Table.2 Per cent heterobeltiosis (BP) and standard heterosis (SC) for grain yield per plant, days to $50 \%$ flowering, days to maturity in pearl millet

\begin{tabular}{|c|c|c|c|c|c|c|c|}
\hline \multirow{2}{*}{$\begin{array}{l}\text { Sr. } \\
\text { No. }\end{array}$} & \multirow[t]{2}{*}{ Crosses } & \multicolumn{2}{|c|}{ Grain yield per plant } & \multicolumn{2}{|c|}{ Days to $50 \%$ flowering } & \multicolumn{2}{|c|}{ Days to maturity } \\
\hline & & $\mathbf{B P}$ & SC & $\mathbf{B P}$ & SC & $\mathbf{B P}$ & SC \\
\hline 1 & JMSA 101 x J-2433 & 10.53 & $21.66 * *$ & -5.44 & 1.23 & -4.76 & -0.41 \\
\hline 2 & JMSA 101 x J-2479 & $36.44 * *$ & $18.53^{*}$ & 0.68 & $8.04 *$ & -0.79 & 3.73 \\
\hline 3 & JMSA 101 x J-2482 & 8.17 & 2.78 & 0.00 & $7.29 *$ & 0.00 & 4.57 \\
\hline 4 & JMSA $101 \times$ J-2496 & 12.00 & -13.55 & -2.72 & 4.38 & -0.40 & 4.15 \\
\hline 5 & JMSA 101 x J-2500 & $-19.20 *$ & $-22.01 * *$ & $-8.16 *$ & -1.47 & $-8.73 *$ & -4.57 \\
\hline 6 & JMSA $101 \times$ J-2503 & $18.26 * *$ & $57.57 * *$ & 3.40 & $10.95 * *$ & 0.79 & 5.39 \\
\hline 7 & JMSA 101 x J-2507 & $22.54 *$ & -1.16 & 6.20 & 0.00 & 4.72 & 1.24 \\
\hline 8 & JMSA $101 \times$ J-2508 & -3.96 & $40.54 * *$ & 3.40 & $10.95 * *$ & 2.38 & $7.06^{*}$ \\
\hline 9 & JMSA $101 \times$ J-2510 & 10.44 & 6.60 & 2.04 & $9.48 * *$ & 0.79 & 5.39 \\
\hline 10 & JMSA 20102 x J-2433 & -12.95 & -4.17 & 4.73 & $13.14 * *$ & 1.17 & $7.88^{*}$ \\
\hline 11 & JMSA 20102 x J-2479 & $97.10 * *$ & $110.04 * *$ & -0.65 & $11.67 * *$ & -1.54 & 6.22 \\
\hline 12 & JMSA 20102 x J-2482 & 14.13 & $21.66 * *$ & 2.03 & $10.23 * *$ & 1.56 & $8.30 *$ \\
\hline 13 & JMSA 20102 x J-2496 & $112.68 * *$ & $126.60 * *$ & 4.70 & $13.86 * *$ & -2.36 & 2.90 \\
\hline 14 & JMSA $20102 \times \mathrm{J}-2500$ & $68.84 * *$ & $79.92 * *$ & 3.97 & $14.61 * *$ & 1.59 & 6.22 \\
\hline 15 & JMSA 20102 x J-2503 & 7.54 & $43.20 * *$ & 0.00 & $11.67 * *$ & -0.38 & $9.12 * *$ \\
\hline 16 & JMSA 20102 x J-2507 & $34.78 * *$ & $43.67 * *$ & $13.95 * *$ & $7.29 *$ & 2.15 & -1.24 \\
\hline 17 & JMSA 20102 x J-2508 & $-41.93 * *$ & $-15.06^{*}$ & 0.00 & $15.33 * *$ & -1.91 & $6.63 *$ \\
\hline 18 & JMSA 20102 x J-2510 & $20.29 *$ & $28.15 * *$ & 0.65 & $12.42 * *$ & 2.27 & $12.04 * *$ \\
\hline 19 & ICMA 841 x J-2433 & $-25.58 * *$ & $-18.07 *$ & 5.41 & $13.86 * *$ & $-10.89 * *$ & -4.98 \\
\hline 20 & ICMA 841 x J-2479 & 11.16 & -3.47 & -1.30 & $10.95 * *$ & -0.38 & $7.47 *$ \\
\hline 21 & ICMA 841 x J-2482 & 9.76 & 4.29 & 4.05 & $12.42 * *$ & -1.17 & 5.39 \\
\hline 22 & ICMA 841 x J-2496 & -4.10 & $-19.69 * *$ & 3.36 & $12.42 * *$ & -0.39 & 4.98 \\
\hline 23 & ICMA 841 x J-2500 & $48.80 * *$ & $43.67 * *$ & -3.31 & $6.57 *$ & $-7.54 *$ & -3.46 \\
\hline 24 & ICMA 841 x J-2503 & $-16.49 *$ & 11.24 & 1.31 & $13.14 * *$ & -4.18 & 4.57 \\
\hline 25 & ICMA 841 x J-2507 & $35.48 * *$ & $13.55 * *$ & $13.95 * *$ & $7.29 *$ & 6.87 & 3.32 \\
\hline 26 & ICMA 841 x J-2508 & $-40.63 * *$ & -13.09 & -5.06 & $9.48 * *$ & -3.05 & 5.39 \\
\hline 27 & ICMA 841 x J-2510 & $24.40 * *$ & $20.04 * *$ & -0.65 & $10.95 * *$ & -3.04 & 5.81 \\
\hline 28 & ICMA 89111 x J-2433 & $33.68 * *$ & $47.14 * *$ & 3.38 & $11.67 * *$ & -0.78 & 5.81 \\
\hline 29 & ICMA 89111 x J-2479 & $20.44 *$ & 4.63 & -1.30 & $10.95 * *$ & -1.94 & 4.98 \\
\hline 30 & ICMA 89111 x J-2482 & 14.63 & 8.92 & 4.05 & $12.42 * *$ & -1.56 & 4.98 \\
\hline 31 & ICMA 89111 x J-2496 & $54.05 * *$ & 10.08 & 0.67 & $9.48 * *$ & -0.39 & 4.98 \\
\hline 32 & ICMA 89111 x J-2500 & -8.00 & -11.24 & -0.66 & $9.48 * *$ & 0.79 & 5.39 \\
\hline 33 & ICMA 89111 x J-2503 & 6.96 & $42.51 * *$ & -0.65 & $10.95 * *$ & 1.16 & $8.30 *$ \\
\hline 34 & ICMA 89111 x J-2507 & $51.67 * *$ & $22.36 * *$ & $11.63 * *$ & 5.10 & 3.43 & 0.00 \\
\hline 35 & ICMA 89111 x J-2508 & $-16.89 * *$ & $21.66^{* * *}$ & -0.65 & $12.42 * *$ & -0.39 & $6.63 *$ \\
\hline 36 & ICMA 89111 x J-2510 & $28.00 * *$ & $23.51 * *$ & -1.31 & $10.23 * *$ & -3.49 & 3.32 \\
\hline 37 & ICMA 04999 x J-2433 & 9.86 & $20.85 * *$ & 3.38 & $11.67 * *$ & 3.70 & 4.57 \\
\hline 38 & ICMA 04999 x J-2479 & $56.02 * *$ & $45.17 * *$ & 5.84 & $18.99 * *$ & 3.70 & 4.57 \\
\hline 39 & ICMA 04999 x J-2482 & 7.72 & 2.32 & 4.05 & $12.42 * *$ & 3.70 & 4.57 \\
\hline 40 & ICMA 04999 x J-2496 & $33.20 * *$ & $23.98 * *$ & 3.36 & $12.42 * *$ & -0.41 & 0.41 \\
\hline 41 & ICMA 04999 x J-2500 & -4.76 & -8.11 & $9.93 * *$ & $21.18 * *$ & 1.65 & 2.49 \\
\hline 42 & ICMA 04999 x J-2503 & $-36.52 * *$ & $-15.41 *$ & 3.92 & $16.05 * *$ & 5.76 & $6.63 *$ \\
\hline 43 & ICMA 04999 x J-2507 & $43.57 * *$ & $33.59 * *$ & $23.26 * *$ & $16.05 * *$ & 1.29 & -2.08 \\
\hline 44 & ICMA 04999 x J-2508 & $-25.30 * *$ & 9.27 & 0.00 & $15.33 * *$ & 4.53 & 5.39 \\
\hline 45 & ICMA 04999 x J-2510 & $-20.40 *$ & $-23.17 * *$ & -5.44 & $17.52 * *$ & -4.76 & 5.39 \\
\hline & S.E.t & \multicolumn{2}{|l|}{0.73} & \multicolumn{2}{|l|}{1.72} & \multicolumn{2}{|l|}{2.99} \\
\hline \multicolumn{2}{|c|}{ Significantly positive crosses } & 19 & 23 & 5 & 40 & 0 & 10 \\
\hline \multicolumn{2}{|c|}{ Significantly negative crosses } & 9 & 6 & 1 & 0 & 3 & 0 \\
\hline
\end{tabular}

$*$, ** were significant at $5 \%$ and $1 \%$ levels of probability, respectively. 
Table.3 Per cent heterobeltiosis (BP) and standard heterosis (SC) for number of nodes on main stem, number of effective tillers per plant and plant height in pearl millet

\begin{tabular}{|c|c|c|c|c|c|c|c|}
\hline \multirow{2}{*}{$\begin{array}{l}\text { Sr. } \\
\text { No. }\end{array}$} & \multirow[t]{2}{*}{ Crosses } & \multicolumn{2}{|c|}{$\begin{array}{l}\text { Number of nodes on } \\
\text { main stem }\end{array}$} & \multicolumn{2}{|c|}{$\begin{array}{l}\text { Number of effective tillers } \\
\text { per plant }\end{array}$} & \multicolumn{2}{|c|}{ Plant height } \\
\hline & & BP & SC & BP & SC & BP & SC \\
\hline 1 & JMSA $101 \times$ J-2433 & $80.56 * *$ & $78.08 * *$ & -5.00 & 11.47 & $41.25 * *$ & $57.91 * *$ \\
\hline 2 & JMSA $101 \times$ J-2479 & $54.17 * *$ & $51.99 * *$ & -13.04 & 17.65 & $49.37 * *$ & $50.27 * *$ \\
\hline 3 & JMSA $101 \times$ J-2482 & $44.44 * *$ & $42.53 * *$ & 8.70 & $46.76 * *$ & $37.42 * *$ & $36.87 * *$ \\
\hline 4 & JMSA 101 x J-2496 & $46.59 * *$ & $76.64 * *$ & -19.23 & 23.82 & $47.38 * *$ & $46.78 * *$ \\
\hline 5 & JMSA $101 \times \mathrm{J}-2500$ & 0.00 & $27.33 *$ & -11.54 & $35.29 *$ & $30.19 * *$ & $36.73 * *$ \\
\hline 6 & JMSA $101 \times \mathrm{J}-2503$ & $34.18 * *$ & $45.21 * *$ & -2.50 & 15.00 & $52.49 * *$ & $51.88 * *$ \\
\hline 7 & JMSA 101 x J-2507 & $51.32 * *$ & $57.53 * *$ & 10.00 & 29.12 & $42.87 * *$ & $42.30 * *$ \\
\hline 8 & JMSA $101 \times \mathrm{J}-2508$ & $26.67 *$ & $30.21 *$ & $50.00 * *$ & $76.76^{* *}$ & $14.40 *$ & $13.94 * *$ \\
\hline 9 & JMSA $101 \times \mathrm{J}-2510$ & 15.85 & $30.21 *$ & 23.81 & $52.94 * *$ & $42.19 * *$ & $41.62 * *$ \\
\hline 10 & JMSA 20102 x J-2433 & 13.25 & $28.77 *$ & -4.00 & $41.47 * *$ & 0.00 & $16.36 * *$ \\
\hline 11 & JMSA 20102 x J-2479 & 15.66 & $31.44^{*}$ & 4.00 & $52.94 * *$ & $21.37 * *$ & $41.22 * *$ \\
\hline 12 & JMSA 20102 x J-2482 & $28.92 * *$ & $46.64 * *$ & $-28.00 *$ & 6.18 & $22.98 * *$ & $43.10 * *$ \\
\hline 13 & JMSA 20102 x J-2496 & -1.14 & $19.11 *$ & 3.85 & $59.12 * *$ & $22.41 * *$ & $42.43 * *$ \\
\hline 14 & JMSA 20102 x J-2500 & $30.11 * *$ & $65.75 * *$ & -11.54 & $35.29 *$ & $15.78 * *$ & $34.72 * *$ \\
\hline 15 & JMSA $20102 \times \mathrm{J}-2503$ & 1.20 & 15.00 & 20.00 & $76.76^{* *}$ & $24.19 * *$ & $44.51 * *$ \\
\hline 16 & JMSA $20102 \times \mathrm{J}-2507$ & $33.73 * *$ & $51.99 * *$ & -8.00 & $35.29 *$ & $15.38 * *$ & $34.25 * *$ \\
\hline 17 & JMSA $20102 \times$ J-2508 & 3.61 & 17.88 & -20.00 & 17.65 & $16.42 * *$ & $35.46 * *$ \\
\hline 18 & JMSA $20102 \times \mathrm{J}-2510$ & 10.84 & $26.10^{*}$ & -4.00 & $41.47 * *$ & $14.75 * *$ & $33.51 * *$ \\
\hline 19 & ICMA 841 x J-2433 & 16.67 & $43.77 * *$ & -4.76 & 17.65 & 0.88 & $14.88 * *$ \\
\hline 20 & ICMA 841 x J-2479 & 4.44 & $28.77^{*}$ & -17.39 & 11.47 & 2.24 & $16.42 * *$ \\
\hline 21 & ICMA 841 x J-2482 & -12.22 & 8.22 & $34.78 * *$ & $82.06 * *$ & $16.60 * *$ & $32.77 * *$ \\
\hline 22 & ICMA 841 x J-2496 & 1.11 & $24.66^{*}$ & $-26.92 *$ & 11.47 & $10.42 *$ & $25.74 * *$ \\
\hline 23 & ICMA 841 x J-2500 & $31.18 * *$ & $67.19 * *$ & $-23.08 *$ & 17.65 & $12.65 *$ & $28.28 * *$ \\
\hline 24 & ICMA 841 x J-2503 & 0.00 & $23.22 *$ & 9.52 & $35.29 *$ & -7.59 & 5.23 \\
\hline 25 & ICMA 841 x J-2507 & 13.33 & $39.66 * *$ & -4.76 & 17.65 & 3.06 & $17.36 * *$ \\
\hline 26 & ICMA 841 x J-2508 & $22.22 *$ & $50.75 * *$ & -4.76 & 17.65 & 4.94 & $19.50 * *$ \\
\hline 27 & ICMA 841 x J-2510 & -15.56 & 4.11 & -4.76 & 17.65 & -0.24 & $13.60 * *$ \\
\hline 28 & ICMA 89111 x J-2433 & $27.14 *$ & $21.99 *$ & 9.09 & $41.47 * *$ & $18.17 * *$ & $32.10 * *$ \\
\hline 29 & ICMA 89111 x J-2479 & 12.86 & 8.22 & -17.39 & 11.47 & $12.30 *$ & $19.98^{* *}$ \\
\hline 30 & ICMA 89111 x J-2482 & $45.07 * *$ & $41.1 * *$ & -13.04 & 17.65 & 9.85 & $17.36 * *$ \\
\hline 31 & ICMA 89111 x J-2496 & $-21.59 *$ & -5.55 & $-23.08 *$ & 17.65 & 5.58 & $12.80 *$ \\
\hline 32 & ICMA 89111 x J-2500 & $-21.51 *$ & 0.00 & $-34.62 * *$ & 0.00 & 9.47 & $16.96 * *$ \\
\hline 33 & ICMA 89111 x J-2503 & $26.58 * *$ & $36.99 * *$ & -13.64 & 11.47 & 9.28 & $16.76^{* *}$ \\
\hline 34 & ICMA 89111 x J-2507 & -10.53 & -6.78 & 0.00 & 29.12 & 3.58 & $10.66^{*}$ \\
\hline 35 & ICMA 89111 x J-2508 & 4.00 & 6.78 & 0.00 & 29.12 & 10.35 & $17.9 * *$ \\
\hline 36 & ICMA 89111 x J-2510 & 4.88 & 17.88 & 9.09 & $41.47 * *$ & 10.10 & $17.62 * *$ \\
\hline 37 & ICMA 04999 x J-2433 & 6.94 & 5.55 & 10.00 & 29.12 & 3.36 & $15.55 * *$ \\
\hline 38 & ICMA 04999 x J-2479 & 11.11 & 9.66 & -4.35 & 29.12 & $23.12 * *$ & $29.56 * *$ \\
\hline 39 & ICMA 04999 x J-2482 & 12.50 & 10.89 & -8.70 & 23.82 & 9.81 & $15.55 * *$ \\
\hline 40 & ICMA 04999 x J-2496 & -9.09 & 9.66 & $42.31 * *$ & $117.35 * *$ & $14.84 *$ & $20.84 * *$ \\
\hline 41 & ICMA 04999 x J-2500 & $-30.11 * *$ & -10.89 & $-30.77 * *$ & 6.18 & 9.55 & $15.28 * *$ \\
\hline 42 & ICMA 04999 x J-2503 & 0.00 & 8.22 & 17.65 & 17.65 & 1.85 & 7.17 \\
\hline 43 & ICMA 04999 x J-2507 & 14.47 & $19.11^{*}$ & 26.32 & $41.47 * *$ & 18.09 & $24.26 * *$ \\
\hline 44 & ICMA 04999 x J-2508 & 8.00 & 10.89 & 16.67 & 23.82 & 8.34 & $14.00 * *$ \\
\hline \multirow[t]{2}{*}{45} & ICMA 04999 x J-2510 & -2.44 & 9.66 & 19.05 & $46.76^{* * *}$ & -3.95 & 1.08 \\
\hline & S.E. \pm & \multicolumn{2}{|l|}{0.51} & \multicolumn{2}{|l|}{0.19} & \multicolumn{2}{|l|}{5.90} \\
\hline \multicolumn{2}{|c|}{ Significantly positive crosses } & 15 & 27 & 3 & 18 & 24 & 42 \\
\hline \multicolumn{2}{|c|}{ Significantly negative crosses } & 3 & 0 & 6 & 0 & 0 & 0 \\
\hline
\end{tabular}

$*$, ** were significant at $5 \%$ and $1 \%$ levels of probability, respectively. 
Table.4 Per cent heterobeltiosis (BP) and standard heterosis (SC) for earhead length, earhead girth and earhead weight in pearl millet

\begin{tabular}{|c|c|c|c|c|c|c|c|}
\hline \multirow{2}{*}{$\begin{array}{l}\text { Sr. } \\
\text { No. }\end{array}$} & \multirow{2}{*}{ Crosses } & \multicolumn{2}{|c|}{ Earhead length } & \multicolumn{2}{|c|}{ Earhead girth } & \multicolumn{2}{|c|}{ Earhead weight } \\
\hline & & BP & SC & BP & SC & $\mathbf{B P}$ & SC \\
\hline 1 & JMSA 101 x J-2433 & $27.12 * *$ & $22.47 * *$ & -6.44 & 11.33 & 9.93 & $55.99 * *$ \\
\hline 2 & JMSA $101 \times$ J-2479 & 1.79 & -7.35 & -7.03 & 10.67 & $66.92 * *$ & $119.26^{* *}$ \\
\hline 3 & JMSA $101 \times$ J-2482 & 12.61 & 5.69 & 0.87 & $20.03 * *$ & -6.86 & 22.29 \\
\hline 4 & JMSA $101 \times$ J-2496 & -6.25 & -8.14 & -5.31 & $15.81 *$ & -16.62 & 9.51 \\
\hline 5 & JMSA $101 \times \mathrm{J}-2500$ & 8.23 & 2.02 & 2.03 & $21.42 * *$ & -12.10 & $57.15^{* *}$ \\
\hline 6 & JMSA $101 \times$ J-2503 & 7.14 & 4.10 & 5.42 & $25.70^{* *}$ & $49.54 * *$ & $96.44 * *$ \\
\hline 7 & JMSA $101 \times$ J-2507 & 8.66 & 2.45 & 5.66 & $25.83^{* *}$ & $35.40 * *$ & $110.39 * *$ \\
\hline 8 & JMSA $101 \times$ J-2508 & $22.87 * *$ & 11.82 & $-17.94 *$ & -2.31 & $25.73 * *$ & $94.44 * *$ \\
\hline 9 & JMSA $101 \times \mathrm{J}-2510$ & 13.90 & 3.67 & 11.81 & $33.08^{* *}$ & -12.44 & $24.40 *$ \\
\hline 10 & JMSA $20102 \times$ x J-2433 & -5.65 & -4.47 & 7.14 & $27.81 * *$ & $-38.41 * *$ & 24.08 \\
\hline 11 & JMSA $20102 \times \mathrm{J}-2479$ & -4.03 & -2.88 & 6.17 & $26.62 * *$ & $-14.09 *$ & $73.10 * *$ \\
\hline 12 & JMSA $20102 \times$ J -2482 & 8.47 & 9.80 & 4.86 & $25.11 * *$ & $-24.89 * *$ & $51.34 * *$ \\
\hline 13 & JMSA 20102 x J-2496 & -2.02 & -0.80 & 8.96 & $33.34 * *$ & $-16.71 *$ & $67.82 * *$ \\
\hline 14 & JMSA $20102 \times \mathrm{J}-2500$ & 2.82 & 4.10 & 4.93 & $25.17 * *$ & $-56.90 * *$ & -13.20 \\
\hline 15 & JMSA $20102 \times \mathrm{J}-2503$ & 12.10 & $13.47 *$ & 12.69 & $34.46 * *$ & $-59.56 * *$ & -18.49 \\
\hline 16 & JMSA $20102 \times \mathrm{J}-2507$ & 2.82 & 4.10 & 7.16 & $27.87 * *$ & $-32.12 * *$ & $36.76^{* * *}$ \\
\hline 17 & JMSA $20102 \times \mathrm{J}-2508$ & $-14.92 *$ & $-13.90^{*}$ & 12.50 & $34.20^{* *}$ & $-24.05 * *$ & $53.03 * *$ \\
\hline 18 & JMSA $20102 \times \mathrm{J}-2510$ & -11.69 & -10.59 & $16.63 *$ & $39.14 * *$ & -11.92 & $77.43 * *$ \\
\hline 19 & ICMA $841 \times$ J-2433 & -7.94 & -5.33 & 5.72 & $32.68 *$ & -5.50 & $51.23 * *$ \\
\hline 20 & ICMA $841 \times$ J J-2479 & 4.76 & 7.78 & 4.50 & $31.17 * *$ & -2.11 & $56.62 * *$ \\
\hline 21 & ICMA 841 x J-2482 & -0.79 & 2.02 & 2.94 & $29.26 * *$ & 8.89 & $74.26 * *$ \\
\hline 22 & ICMA $841 \times$ J-2496 & -3.97 & -1.22 & 12.74 & $41.51 *$ & $-33.10 * *$ & 7.08 \\
\hline 23 & ICMA $841 \times$ J-2500 & -11.11 & -8.57 & 11.83 & $40.39 * *$ & $-37.59 * *$ & 11.51 \\
\hline 24 & ICMA $841 \times$ J-2503 & -5.56 & -2.88 & 1.84 & $27.81 * *$ & $24.74 * *$ & $99.61 * *$ \\
\hline 25 & ICMA $841 \times$ J-2507 & -7.94 & -5.33 & 6.46 & $33.61 * *$ & -15.54 & $35.18 * *$ \\
\hline 26 & ICMA $841 \times$ J-2508 & 5.16 & 8.14 & 0.87 & $26.62 * *$ & $-33.49 * *$ & 6.44 \\
\hline 27 & ICMA $841 \times$ J-2510 & -5.16 & -2.45 & 8.08 & $35.65^{* *}$ & -5.90 & $50.60 * *$ \\
\hline 28 & ICMA 89111 x J-2433 & 4.47 & 4.90 & -2.85 & $27.87 * *$ & $20.96^{*}$ & $98.35^{* *}$ \\
\hline 29 & ICMA $89111 \times$ J-2479 & 0.41 & 0.80 & -1.62 & $29.45^{* *}$ & 5.11 & $72.36^{* *}$ \\
\hline 30 & ICMA 89111 x J-2482 & 6.91 & 7.35 & 9.16 & $43.69^{* *}$ & 14.09 & $87.04 * *$ \\
\hline 31 & ICMA 89111 x J-2496 & 0.41 & 0.80 & -4.62 & 25.50 ** & -1.63 & $61.27 * *$ \\
\hline 32 & ICMA $89111 \times$ J-2500 & -2.44 & -2.02 & -10.30 & $18.05^{*}$ & 1.26 & $81.02 * *$ \\
\hline 33 & ICMA $89111 \times$ J-2503 & 3.66 & 4.10 & -4.87 & $25.17 * *$ & 12.50 & $84.40 * *$ \\
\hline 34 & ICMA $89111 \times$ J-2507 & 2.03 & 2.45 & 5.49 & $38.81^{* *}$ & $39.35 * *$ & $128.45^{* *}$ \\
\hline 35 & ICMA $89111 \times$ J-2508 & -1.22 & -0.80 & $-13.99 *$ & 13.24 & 3.31 & $69.4 * *$ \\
\hline 36 & ICMA $89111 \times$ J-2510 & 1.63 & 2.02 & -7.34 & $21.94 * *$ & $23.71 * *$ & $102.78^{* *}$ \\
\hline 37 & ICMA $04999 \times$ J-2433 & 13.56 & 9.37 & -10.89 & 13.57 & $-46.55 * *$ & $26.94 *$ \\
\hline 38 & ICMA $04999 \times$ J-2479 & 5.08 & 1.22 & -3.74 & $22.73^{* *}$ & $-13.61 *$ & $105.21^{* *}$ \\
\hline 39 & ICMA 04999 x J-2482 & 9.32 & 5.33 & $-12.94 *$ & 11.00 & $-37.21 * *$ & $49.12 * *$ \\
\hline 40 & ICMA $04999 \times$ J-2496 & $16.67 *$ & $14.27 *$ & -0.53 & $26.82 * *$ & -10.61 & $112.29 * *$ \\
\hline 41 & ICMA $04999 \times \mathrm{J}-2500$ & 1.69 & -2.02 & -7.53 & $17.86^{*}$ & $-35.75^{* *}$ & $52.61 * *$ \\
\hline 42 & ICMA $04999 \times \mathrm{J}-2503$ & 0.42 & -2.45 & $-13.08^{*}$ & 10.81 & $-43.43 * *$ & $34.33 * *$ \\
\hline 43 & ICMA $04999 \times$ J-2507 & 5.51 & 1.65 & -10.48 & $14.10^{*}$ & $-27.33 * *$ & $72.57 * *$ \\
\hline 44 & ICMA $04999 \times \mathrm{J}-2508$ & 2.54 & -1.22 & 0.36 & $27.94 * *$ & $-13.82 *$ & $104.68 * *$ \\
\hline 45 & ICMA $04999 \times \mathrm{J}-2510$ & 3.81 & 0.00 & -9.05 & $15.95^{*}$ & $-32.08 * *$ & $61.37 * *$ \\
\hline & S.E. \pm & \multicolumn{2}{|l|}{1.20} & \multicolumn{2}{|l|}{1.23} & \multicolumn{2}{|l|}{1.34} \\
\hline \multicolumn{2}{|c|}{ Significantly positive crosses } & 3 & 3 & 1 & 38 & 8 & 37 \\
\hline \multicolumn{2}{|c|}{ Significantly negative crosses } & 1 & 1 & 4 & 0 & 19 & 0 \\
\hline
\end{tabular}

$*, * *$ were significant at $5 \%$ and $1 \%$ levels of probability, respectively. 
Table.5 Per cent heterobeltiosis (BP) and standard heterosis (SC) for test weight and panicle index in pearl millet

\begin{tabular}{|c|c|c|c|c|c|}
\hline & \multirow[t]{2}{*}{ Crosses } & \multicolumn{2}{|l|}{ Test weight } & \multicolumn{2}{|c|}{ Panicle index } \\
\hline & & BP & SC & BP & SC \\
\hline 1 & JMSA 101 x J-2433 & $8.85 * *$ & $19.99 * *$ & $-24.49 *$ & $28.34 *$ \\
\hline 2 & JMSA 101 x J-2479 & $24.25 * *$ & $39.59 * *$ & 9.20 & $85.60 * *$ \\
\hline 3 & JMSA 101 x J-2482 & $-9.42 * *$ & $9.54 * *$ & $-29.80 * *$ & 19.31 \\
\hline 4 & JMSA 101 x J-2496 & $-10.58 * *$ & $21.02 * *$ & $-31.31 * *$ & 26.7 \\
\hline 5 & JMSA $101 \times$ J-2500 & $12.78 * *$ & $23.34 * *$ & 9.74 & $103.63 * *$ \\
\hline 6 & JMSA $101 \times \mathrm{J}-2503$ & $25.29 * *$ & $35.72 * *$ & $-26.52 * *$ & 24.89 \\
\hline 7 & JMSA $101 \times$ J-2507 & $7.15 *$ & $16.51 * *$ & 10.39 & $112.62 * *$ \\
\hline 8 & JMSA $101 \times$ J-2508 & $10.92 * *$ & $20.12 * *$ & -18.41 & $38.69 * *$ \\
\hline 9 & JMSA $101 \times$ J-2510 & $-16.41 * *$ & $24.50 * *$ & $-31.26 * *$ & 16.84 \\
\hline 10 & JMSA $20102 \times$ J-2433 & $-8.31 * *$ & $24.24 * *$ & $-31.63 * *$ & $29.36^{*}$ \\
\hline 11 & JMSA $20102 \times$ J-2479 & $-13.39 * *$ & $17.28 * *$ & $-56.61 * *$ & -17.91 \\
\hline 12 & JMSA 20102 x J-2482 & $-18.18 * *$ & $10.83 * *$ & $-34.09 * *$ & 24.69 \\
\hline 13 & JMSA 20102 x J-2496 & $-11.20 * *$ & $20.25 * *$ & $-60.75 * *$ & -25.75 \\
\hline 14 & JMSA $20102 \times \mathrm{J}-2500$ & $-13.45 * *$ & $17.28 * *$ & $-74.55 * *$ & $-51.85 * *$ \\
\hline 15 & JMSA $20102 \times$ J-2503 & $-8.47 * *$ & $23.98 * *$ & $-70.00 * *$ & $-43.24 * *$ \\
\hline 16 & JMSA 20102 x J-2507 & $-19.32 * *$ & $9.28 * *$ & $-50.46 * *$ & -4.57 \\
\hline 17 & JMSA 20102 x J-2508 & $-15.51 * *$ & $14.44 * *$ & -4.62 & $80.45 * *$ \\
\hline 18 & JMSA 20102 x J-2510 & $-13.12 * *$ & $29.53 * *$ & $-26.75 * *$ & $38.57 * *$ \\
\hline 19 & ICMA 841 x J-2433 & $-10.31 * *$ & $34.3 * *$ & -3.58 & $84.74 * *$ \\
\hline 20 & ICMA 841 x J-2479 & $-19.12 * *$ & $21.15^{* *}$ & -14.18 & $64.43 * *$ \\
\hline 21 & ICMA 841 x J-2482 & $-16.88 * *$ & $24.50 * *$ & -10.35 & $71.77 * *$ \\
\hline 22 & ICMA 841 x J-2496 & $-27.91 * *$ & $7.87 * *$ & $-28.04 * *$ & $37.88 * *$ \\
\hline 23 & ICMA 841 x J-2500 & $-26.41 * *$ & $10.19 * *$ & $-58.25 * *$ & -20.01 \\
\hline 24 & ICMA 841 x J-2503 & $-21.10 * *$ & $18.05 * *$ & -6.27 & $79.57 * *$ \\
\hline 25 & ICMA 841 x J-2507 & $-24.52 * *$ & $13.02 * *$ & $-37.69 * *$ & 20.01 \\
\hline 26 & ICMA 841 x J-2508 & $-34.37 * *$ & -1.81 & $-33.23 * *$ & $27.93^{*}$ \\
\hline 27 & ICMA 841 x J-2510 & $-4.31 *$ & $43.33 * *$ & $-33.97 * *$ & 26.52 \\
\hline 28 & ICMA 89111 x J-2433 & $-27.03 * *$ & $6.58 * *$ & $-41.64 * *$ & $35.95 *$ \\
\hline 29 & ICMA 89111 x J-2479 & $-17.34 * *$ & $20.63 * *$ & $-27.20 * *$ & $69.59 * *$ \\
\hline 30 & ICMA 89111 x J-2482 & $-17.26 * *$ & $20.76 * *$ & $-25.74 * *$ & $73.00 * *$ \\
\hline 31 & ICMA 89111 x J-2496 & $-16.11 * *$ & $22.44 * *$ & $-36.98 * *$ & $46.81 * *$ \\
\hline 32 & ICMA 89111 x J-2500 & $-25.41 * *$ & $8.90 *$ & -11.10 & $107.11 * *$ \\
\hline 33 & ICMA 89111 x J-2503 & $-15.96 * *$ & $22.70 * *$ & $-44.33 * *$ & $29.68 *$ \\
\hline 34 & ICMA 89111 x J-2507 & $-21.88 * *$ & $14.06 * *$ & $-19.38 * *$ & $87.81 * *$ \\
\hline 35 & ICMA 89111 x J-2508 & $-27.18 * *$ & $6.32 * *$ & $-40.39 * *$ & $38.88 * *$ \\
\hline 36 & ICMA 89111 x J-2510 & $-27.69 * *$ & $7.74 * *$ & $-28.81 * *$ & $65.84 * *$ \\
\hline 37 & ICMA 04999 x J-2433 & $-23.52 * *$ & 2.19 & $-58.77 * *$ & 5.53 \\
\hline 38 & ICMA 04999 x J-2479 & -0.84 & $32.50 * *$ & $-44.62 * *$ & $41.74 * *$ \\
\hline 39 & ICMA 04999 x J-2482 & $-12.68 * *$ & $16.63 * *$ & $-42.17 * *$ & $48.04 * *$ \\
\hline 40 & ICMA 04999 x J-2496 & $-20.17 * *$ & $7.99 * *$ & $-32.82 * *$ & $71.96 * *$ \\
\hline 41 & ICMA 04999 x J-2500 & $-10.88 * *$ & $19.08 * *$ & $-35.13 * *$ & $66.05 * *$ \\
\hline 42 & ICMA 04999 x J-2503 & $-9.20 * *$ & $21.28 * *$ & $-37.67 * *$ & $59.55 * *$ \\
\hline 43 & ICMA 04999 x J-2507 & $-15.83 * *$ & $12.38 * *$ & $-49.44 * *$ & $29.42 *$ \\
\hline 44 & ICMA 04999 x J-2508 & $-16.18 * *$ & $11.99 * *$ & $-26.64 * *$ & $87.78 * *$ \\
\hline 45 & ICMA 04999 x J-2510 & $-28.79 * *$ & $6.06^{*}$ & $-17.62 * *$ & $110.86 * *$ \\
\hline \multicolumn{2}{|c|}{ S.E. \pm} & \multicolumn{2}{|l|}{0.24} & \multicolumn{2}{|l|}{17.33} \\
\hline \multicolumn{2}{|c|}{ Significantly positive crosses } & 6 & 43 & 0 & 31 \\
\hline \multicolumn{2}{|c|}{ Significantly negative crosses } & 38 & 0 & 35 & 2 \\
\hline
\end{tabular}

$*$, ** were significant at $5 \%$ and $1 \%$ levels of probability, respectively. 
Table.6 Per cent heterobeltiosis (BP) and standard heterosis (SC) for total biomass per plant and harvest index in pearl millet

\begin{tabular}{|c|c|c|c|c|c|}
\hline \multirow[t]{2}{*}{ Sr. no. } & \multirow[t]{2}{*}{ Crosses } & \multicolumn{2}{|c|}{ Total biomass per plant } & \multicolumn{2}{|l|}{ Harvest index } \\
\hline & & $\mathbf{B P}$ & SC & BP & SC \\
\hline 1 & JMSA $101 \times$ J-2433 & $-20.17 *$ & $37.66 * *$ & $29.80 * *$ & $-11.68 *$ \\
\hline 2 & JMSA 101 x J-2479 & -4.20 & $114.84 * *$ & 23.16 & $-44.83 * *$ \\
\hline 3 & JMSA 101 x J-2482 & $31.92 * *$ & $155.92 * *$ & -16.10 & $-58.94 * *$ \\
\hline 4 & JMSA 101 x J-2496 & $25.43 * *$ & $121.58 * *$ & -10.43 & $-59.88 * *$ \\
\hline 5 & JMSA $101 \times$ J-2500 & $69.96 * *$ & $193.10 * *$ & $-70.61 * *$ & $-73.34 * *$ \\
\hline 6 & JMSA $101 \times$ J-2503 & $28.78 * *$ & $122.12 * *$ & $-26.43 * *$ & $-29.06 * *$ \\
\hline 7 & JMSA $101 \times$ J-2507 & $70.80 * *$ & $194.57 * *$ & $-41.78 * *$ & $-66.35 * *$ \\
\hline 8 & JMSA $101 \times$ J-2508 & $36.01 * *$ & $134.57 * *$ & $-53.26 * *$ & $-39.89 * *$ \\
\hline 9 & JMSA 101 x J-2510 & $95.38 * *$ & $236.96 * *$ & $-59.20 * *$ & $-68.38 * *$ \\
\hline 10 & JMSA $20102 \times \mathrm{J}-2433$ & $-33.23 * *$ & $115.05 * *$ & $-34.53 * *$ & $-55.44 * *$ \\
\hline 11 & JMSA $20102 \times$ J-2479 & $-22.11 * *$ & $150.87 * *$ & $116.06 * *$ & $-16.22 * *$ \\
\hline 12 & JMSA $20102 \times$ J-2482 & $-50.94 * *$ & $57.99 * *$ & $57.22 * *$ & $-23.03 * *$ \\
\hline 13 & JMSA $20102 \times$ J-2496 & $-10.12 *$ & $189.51 * *$ & $114.16 * *$ & $-21.71 * *$ \\
\hline 14 & JMSA $20102 \times \mathrm{J}-2500$ & $9.45 *$ & $252.55 * *$ & $-43.77 * *$ & $-48.99 * *$ \\
\hline 15 & JMSA $20102 \times \mathrm{J}-2503$ & 4.27 & $235.87 * *$ & $-55.71 * *$ & $-57.30 * *$ \\
\hline 16 & JMSA $20102 \times$ J-2507 & $-36.06 * *$ & $105.92 * *$ & $34.37 * *$ & $-22.37 * *$ \\
\hline 17 & JMSA $20102 \times \mathrm{J}-2508$ & $-36.30 * *$ & $105.16 * *$ & $-66.60 * *$ & $-57.06 * *$ \\
\hline 18 & JMSA $20102 \times \mathrm{J}-2510$ & $-13.98 * *$ & $177.07 * *$ & $-40.32 * *$ & $-53.74 * *$ \\
\hline 19 & ICMA 841 x J-2433 & -6.52 & $105.60 * *$ & $-41.43 * *$ & $-60.13 * *$ \\
\hline 20 & ICMA 841 x J-2479 & $-38.53 * *$ & $37.88 * *$ & $81.01 * *$ & $-29.81 * *$ \\
\hline 21 & ICMA 841 x J-2482 & $52.14 * *$ & $234.62 * *$ & $-36.14 * *$ & $-68.74 * *$ \\
\hline 22 & ICMA 841 x J-2496 & $-39.79 * *$ & $32.45 *$ & $58.32 * *$ & $-39.72 * *$ \\
\hline 23 & ICMA 841 x J-2500 & $38.22 * *$ & $203.97 * *$ & $-47.85 * *$ & $-52.67 * *$ \\
\hline 24 & ICMA 841 x J-2503 & $17.99 * *$ & $159.51 * *$ & $-55.57 * *$ & $-57.15 * *$ \\
\hline 25 & ICMA 841 x J-2507 & 8.07 & $137.66 * *$ & -17.43 & $-52.29 * *$ \\
\hline 26 & ICMA 841 x J-2508 & -6.05 & $106.63 * *$ & $-66.83 * *$ & $-57.34 * *$ \\
\hline 27 & ICMA 841 x J-2510 & $-19.77 * *$ & $76.47 * *$ & -11.45 & $-31.34 * *$ \\
\hline 28 & ICMA 89111 x J-2433 & $15.36 *$ & $171.36 * *$ & $-20.26 *$ & $-45.73 * *$ \\
\hline 29 & ICMA 89111 x J-2479 & -5.69 & $121.85 * *$ & 21.98 & $-52.70 * *$ \\
\hline 30 & ICMA 89111 x J-2482 & -5.58 & $122.12 * *$ & 0.14 & $-50.97 * *$ \\
\hline 31 & ICMA 89111 x J-2496 & -10.36 & $110.87 * *$ & $42.92 *$ & $-47.75 * *$ \\
\hline 32 & ICMA 89111 x J-2500 & $-24.37 * *$ & $77.88 * *$ & $-45.11 * *$ & $-50.20 * *$ \\
\hline 33 & ICMA 89111 x J-2503 & -10.67 & $110.16 * *$ & $-29.68 * *$ & $-32.20 * *$ \\
\hline 34 & ICMA 89111 x J-2507 & -1.12 & $132.61 * *$ & -8.95 & $-47.39 * *$ \\
\hline 35 & ICMA 89111 x J-2508 & $-19.57 * *$ & $89.18^{* * *}$ & $-50.06 * *$ & $-35.78 * *$ \\
\hline 36 & ICMA 89111 x J-2510 & -2.96 & $128.26 * *$ & $-30.18 * *$ & $-45.88 * *$ \\
\hline 37 & ICMA 04999 x J-2433 & -3.24 & $110.00 * *$ & -15.44 & $-42.47 * *$ \\
\hline 38 & ICMA 04999 x J-2479 & -8.34 & $105.54 * *$ & $64.98 * *$ & $-29.30 * *$ \\
\hline 39 & ICMA 04999 x J-2482 & $-40.28 * *$ & $29.62 *$ & $61.83 * *$ & $-20.78 * *$ \\
\hline 40 & ICMA 04999 x J-2496 & $66.11 * *$ & $260.49 * *$ & -19.77 & $-65.63 * *$ \\
\hline 41 & ICMA 04999 x J-2500 & $-19.15 * *$ & $75.49 * *$ & $-42.19 * *$ & $-47.54 * *$ \\
\hline 42 & ICMA 04999 x J-2503 & $16.69 *$ & $153.26 * *$ & $-65.29 * *$ & $-66.53 * *$ \\
\hline 43 & ICMA 04999 x J-2507 & $48.18 * *$ & $221.58 * *$ & $-28.11 * *$ & $-58.47 * *$ \\
\hline 44 & ICMA 04999 x J-2508 & 5.51 & $128.97 * *$ & $-62.88 * *$ & $-52.27 * *$ \\
\hline 45 & ICMA 04999 x J-2510 & -9.85 & $95.65 * *$ & $-49.28 * *$ & $-60.69 * *$ \\
\hline \multicolumn{2}{|l|}{ S.E.t } & \multicolumn{2}{|l|}{2.64} & \multicolumn{2}{|l|}{2.87} \\
\hline \multicolumn{2}{|c|}{ Significantly positive crosses } & 15 & 45 & 10 & 0 \\
\hline \multicolumn{2}{|c|}{ Significantly negative crosses } & 15 & 0 & 25 & 45 \\
\hline
\end{tabular}

$*$, ** were significant at $5 \%$ and $1 \%$ levels of probability, respectively. 
The results revealed that the best three hybrids identified on the basis of per se performance and standard heterosis for seed yield per plant viz., JMSA 20102 x J-2496, JMSA 20102 x J-2479 and ICMA 841 x J2500 also depicted the significant positive standard heterosis over GHB 732 for important yield contributing traits like

On the basis of per se performance of hybrids (Table 1) for grain yield per plant, the crosses JMSA 20102 x J-2496, JMSA 20102 x J-2479 and JMSA $20102 \times \mathrm{J}-2500$ were found the superior among forty five hybrids. The second highest cross combination JMSA 20102 x J2479 also recorded higher per se performance for harvest index.

The third ranked cross JMSA 20102 x J-2500 notice good performer for total biomass per plant. This suggested that the parents involved in these hybrids had a strong tendency to transmit the higher gain to the off springs. Valu (2006), Davada et al., (2008), Chotaliya et al., (2009), Dangaria et al.,(2009), Vagadiya et al., (2010a), Jethava et al., (2012) and Bhadalia et al., (2013) were also akin to this observation.

The estimates of heterobeltiosis for this important trait like harvest index varied from -70.61 to 116.06 per cent. Among the 45 crosses, ten crosses demonstrated positively significant heterobeltiosis.

The cross showed the highest heterobeltiosis was JMSA 20102 x J-2479 (116.06\%) followed by JMSA 20102 x J-2496 (114.16\%) and ICMA $841 \times \mathrm{J}-2479$ (81.01\%). The minimum heterobeltiosis for this trait was noticed by cross JMSA 101 x J-2500 ($70.61 \%)$.

On the whole, considerable heterobeltiosis and standard heterosis observed for seed yield and other associated characters suggested the presence of large genetic diversity among the parents and also unidirectional distribution of allelic constitution contributing towards desirable heterosis in the present material. The moderate to low magnitude of desirable heterosis observed for days to $50 \%$ flowering, Days to maturity indicated the narrow genetic base among the parents.

The best two promising hybrids namely JSMA 20102 x J-2496 and JSMA 20102 x J2479exhibited the highest per se performance, positively significant and high magnitude of heterobeltiosis as well as standard heterosis and highly significant sca effects for grain yield per plant.

These hybrids also registered significantly higher heterosis and sca effects in desired direction for other yield attributing components. Therefore, these hybrids could be further evaluated over years and locations to exploit for commercial cultivation.

\section{References}

Andrews, D. J. and Anand, K. K. 1992. Pearl millet for food, feed and forage. Advances in Agronomy, 48: 89-139.

Arulselvi, S., Mohanasundaram, K., Selvi, B. and Malarvizhi, P. 2006. Heterosis for grain yield components and grain quality characters in pearl millet. ISMN., 47: 36-38.

Athwal, D. S. 1965. Hybrid bajra-1 marks a new era. Ind. Farming, 15: 6-7.

Bhadalia, A. S., Dhedhi, K. K. and Joshi, H. J. 2013. Heterosis studies in diallel crosses of pearl millet. J. Agric. Res. Tech., 38(3): 360-365.

Burton, G. W. 1965. Pearl millet tift 23A released. Crops \& Soil, 17: 19.

Burton, G. W. 1983. Breeding pearl millet. Plant Breed. Rev., 1: 162-182.

Chotaliya, J. M., Dangaria, C. J. and Dhedhi, K. K. 2009. Exploitation of heterosis and selection of superior inbreds in pearl millet. Int. J. Agric. Sci., 5(2): 531-535.

Dangaria, C. J., Chotalia, J. M., Savaliya, J. J., 
Davda, B. K. and Pansuriya, A. G. 2009 b. Combining ability analysis in pearl millet [Pennisetum glaucum (L.) R. Br.]. Agric. Sci. Digest, 29(4): 287-290.

Davda, B. K., Dhedhi, K. K., Dangaria, C. J. and Joshi, A. K. 2008. Heterosis for grain yield and its components in pearl millet. Int. J. Agric. Sci., 4(1): 371-376.

Fonseca, S. and Patterson, F. L. 1968. Hybrid vigour in a seven parent diallel cross in common winter wheat [Triticum aestivum L.]. Crop Sci., 8: 85-88.

Jethava, A. S., RavalLata; Madariya, R. B., Mehta, D. R. and Mandavia, Chetana. 2012. Heterosis for grain yield and its related characters in pearl millet. Elect. J. Plant Breed., 3(3): 848-852.

Meredith, W. R. and Bridge, R. R. 1972. Heterosis and gene action in cotton $[G$. hirsutum L.]. Crop Sci., 12: 304-10.

Pal, M., Daka, J. and Rai, R. K. 1996. Fundamentals of Cereal Crop Production. Tata Mc Grow Hill Publishing Company Limited, New Delhi.

Panse, V. G. and Sukhatme, P. V. 1985. Statistical methods for agricultural workers, Indian council of Agricultural Research, New Delhi.

Patel, K. Y., Kulkarni, G. U. and Patel, D. R. 2008. Heterosis and combining ability studies for fodder yield and its components in pearl millet. J. Maharashtra Agric. Univ., 33(1): 9-12.

Patel, S. M. 2012. Genetic analysis of grain yield and its component characters in pearl millet (Pennisetum glaucum (L.) R. Br.), Unpublished M. Sc. (Agri.) thesis submitted to Anand Agricultural University, Anand.

Patel, T. K. 2014. Studies on heterotic effects, gene effects and stability parameters for grain yield, its components and quality characters in pearl millet (Pennisetum glaucum (L.) R. Br.). Unpublished Ph.D. (Agri.) thesis submitted to Anand Agricultural University, Anand.

Ramamoorthi, N and Govindasaru, R. 2000. Heterosis for grain yield and its components in pearl millet (Pennisetum glaucum (L.) R. Br.). Madras Agric. J., 87(1-3): 159-161.

Shah, K. A. 2009. Genetic analysis of yield and grain quality traits in pearl millet [Pennisetum glaucum (L.) R. Br.] Unpublished Ph.D. thesis submitted to Anand Agricultural University, Anand.

Umaretiya, S. V. 2006. Line $x$ tester studies on heterosis, gene action and phenotypic stability in pearl millet [Pennisetum glaucum (L.) R. Br.], Unpublished M.Sc. (Agri.) thesis submitted to Junagadh Agricultural University, Junagadh.

Vagadiya, K. J., Dhedhi, K. K., Joshi, H. J., Vekariya, H. B. and Bhadalia, A. S. 2010a. Heterosis studies for grain yield and its components in pearl millet. Int. J. Agric. Sci., 6(2): 625-629.

Vagadiya, K. J., Dhedhi, K. K., Joshi, H. J., Vekariya, H. B. and Bhadalia, A. S. 2010b. Genetic architecture of grain yield and its components in pearl millet. Int. J. Plant Sci., 5(2): 582-586.

Valu, N. G. 2006. Combining ability in pearl millet [Pennisetum glaucum (L.) R. Br.]. Unpublished M.Sc. (Agri.) thesis submitted to Junagadh Agricultural University, Junagadh.

Vetriventhan, M., Nirmalakumari, A. and Ganapathy, S. 2008. Heterosis for grain yield components in pearl millet. World. $J$. Agric. Sci., 4(5): 657-660.

\section{How to cite this article:}

Zeal R. Acharya, M.D. Khanapara, V.B. Chaudhari and Jalpa D. Dobaria. 2017. Exploitation of Heterosis in Pearl Millet [Pennisetum glaucum (L.) R. Br.] for Yield and its Component Traits by Using Male Sterile Line. Int.J.Curr.Microbiol.App.Sci. 6(12): 750-759. doi: https://doi.org/10.20546/ijcmas.2017.612.079 